POS $\quad$ PROCEEDINGS

\title{
Resolving discrepancy in the pPN OH 231.8+4.2
}

\section{J.-F. Desmurs*}

Observatorio Astronómico Nacional, Spain

E-mail: desmurseon.es

\section{J. Alcolea}

Observatorio Astronómico Nacional, Spain

E-mail: j.alcolea@oan.es

\section{Lindqvist}

Department of Space, Earth and Environment, Chalmers University of Technology, Onsala Space Observatory, 43992 Onsala, Sweden

E-mail: michael.lindqvist@chalmers.se

\section{Bujarrabal}

Observatorio Astronómico Nacional, Spain

E-mail: v.bujarrabal@oan.es

\section{R. Soria-Ruiz}

Observatorio Astronómico Nacional, Spain

E-mail: r.soria@oan.es

\section{P. de Vicente}

Observatorio de Yebes (IGN), Yebes, Spain

E-mail: p.devicentedoan.es

$\mathrm{OH} 231.8+4.2$ is an archetypal pre-planetary nebulae (pPN). It's a binary system surrounded by bipolar nebula. Some years ago the authors extensively studied it and performed several VLBI observations from which they obtained mas-resolution maps of the $\mathrm{SiO}(7 \mathrm{~mm})$ and $\mathrm{H}_{2} \mathrm{O}(1.3 \mathrm{~cm})$ maser emissions (see [7]). $\mathrm{H}_{2} \mathrm{O}$ masers were found to be distributed in two areas along the symmetry axis of the nebulae oriented nearly north-south delineating a bipolar outflow and their astrometric positions were accurately measured. $\mathrm{SiO}$ masers, indicating the position of the Mira component of the binary system, form a structure perpendicular to the axis of the nebulae. The general picture of the source looked satisfactory, except for the relative position of the two masers. Surprisingly, SiO masers, were tentatively placed 250 mas away (370 AU) from the apparent center of the outflow. Using the ALMA we observed the $\mathrm{SiO}$ maser emission at $86 \mathrm{GHz}$ and accurately derived the position of the Mira component. Combining our previous VLBA data and our new ALMA observations we are now able to give a more complete and detailed description of the inner part of this amazing $\mathrm{pPN}$.

14th European VLBI Network Symposium \& Users Meeting (EVN 2018)

8-11 October 2018

Granada, Spain

\footnotetext{
*Speaker.
} 


\section{Introduction}

Planetary nebulae (PNe) evolve from the envelopes of asymptotic giant branch (AGB) stars, through the very short $(\sim 1000 \mathrm{yr})$ phase of pre-planetary nebula $(\mathrm{pPN})$. During this phase, the nebular morphology and kinematics are dramatically altered: the spherical, slowly expanding AGB envelope becomes a PN, usually with axial symmetry and high axial velocities. This spectacular metamorphosis is thought to result from the interaction of a fast and highly collimated bipolar wind, ejected in the late-AGB or early post-AGB phase, with the slow spherical AGB envelope. To explain the onset of these bipolar flows several models have postulated the presence of binary companions, an accretion disk, rotating magnetic fields or a combination of these (see e.g. [2]; [20]; [11]). A spectacular case of a pPN is the Rotten Egg Nebula, a.k.a. $\mathrm{OH} 231.8+4.2$, a 55" long bi-lobed nebula around the binary star QX Pup. OH 231.8+4.2 (hereafter OH 231.8) is a well studied pPN over a wide range of wavelengths. The distance to this source, $1.54+0.02-0.01 \mathrm{kpc}$ ([5]), and the inclination of the bipolar axis with respect to the plane of the sky, $\sim 36^{\circ}$, are well known. The central source is a binary system formed by the primary, a M9 III Mira variable (i.e. AGB star), and an A0 main sequence companion (see [18]). The presence of a late-type star in the core of a bipolar post-AGB nebula like $\mathrm{OH} 231.8$ is very unusual since the central stars of pPNe are typically hotter with spectral types from A to K. This led some authors to suggest that this could be a born-again object: a star that after having initiated its post-AGB evolution, comes back to the AGB as a result of the last thermal pulse (see [1] and references therein). This remarkable bipolar nebula shows all the signs of post-AGB evolution: fast bipolar outflows with velocities $\sim 200-400 \mathrm{~km} \mathrm{~s}^{-1}$, shock-excited gas and shock-induced chemistry (see [16]; [1]; [3]; [4]; [19]). In spite of this, $\mathrm{OH} 231.8$ also presents $\mathrm{SiO}$ and $\mathrm{H}_{2} \mathrm{O}$ maser emission, which can be used to probe the very inner environment of this pPN with the highest resolution.

Although the central sources remains directly unseen, we know that it is a binary system, as the scattered light shows the presence a cool M9III Mira variable primary and a weaker A0V secondary (see [18]). There is also evidence for various sites of activity, as shown by the misalignment between the symmetry axis of $\mathrm{CO}$ and that of other images (see [12]), and also by the 0,25 .' separation tentatively found between the locations of the $\mathrm{SiO}$ (see [16]) and $22 \mathrm{GHz} \mathrm{H}_{2} \mathrm{O}$ masers (see [5]). It has been suggested that the presence of yet another star could explain the difference in size between the north and south lobes: if there is another mass-losing AGB star close to the engine of $\mathrm{OH} 231.8$, this would introduce an asymmetry in the ambient medium the post-AGB outflow is running into ([15]). However the exact location of these different sources and their role in the post-AGB evolution of the system remains unclear, mostly due to the lack of sub-arc second resolution images of the molecular gas in the central parts of the nebula.

About 15 years ago, we carried out several VLBA projects to study the maser emission from this source. We observed the $7 \mathrm{~mm} \mathrm{SiO}$ maser $v=2, J=1-0$ emission ([17]) and obtained 0.3 masresolution VLBA maps of the $3 \mathrm{~mm} \mathrm{SiO}$ maser $v=1, J=2-1$ emission (see [7]). However, we were not able to determine an accurate absolute position for the $\mathrm{SiO}$ emission with subsequent phase referencing VLBI observations.

Our observations revealed that the $\mathrm{SiO}$ emission arises from several spots, less than $\sim 10^{13} \mathrm{~cm}$ in size, forming a structure elongated in a direction perpendicular to the symmetry axis of the nebula occupying a region of about 8 mas $(12 \mathrm{AU})$ in extension and a few mas across. Such a 
different distribution from the spherical shells of spots usually found in evolved stars (see e.g. VLBA observations by [9]; [6]), seems to reveal the presence of an equatorial torus of gas around the primary Mira star with a radius of $\sim 6 \mathrm{AU}$. The velocities found along the torus are consistent with the keplerian rotation around the central star(s) ([17]).

In addition we also mapped the $\mathrm{H}_{2} \mathrm{O}$ maser emission which was found to arise from several compact spots distributed in mainly two areas about 30 mas each in size (see Fig. 2, [8]) and separated by about 60 mas in the direction of the symmetry axis of the nebula. Their absolute positions and the proper motions of the individual spots were measured very accurately. The northern area corresponds to the blue-shifted emission and the southern area to the red-shifted one. The region in which the water masers are distributed seems to delineate the walls of a double cone outflow perpendicular to the equatorial disk traced by the $\mathrm{SiO}$ masers.

The emerging picture seems consistent for the results of the relative position of the two maser emissions. The published results on the $\mathrm{SiO}$ emission ([17]), tentatively place the Mira primary about 250 mas away from the center of the $\mathrm{H}_{2} \mathrm{O}$ outflow. This separation results in a lower limit distance between the two centers of maser emission at least $370 \mathrm{AU}$, far too much for an interacting binary system.

During the last 20 years the $\mathrm{SiO}$ masers have been slowly fading away. At the epoch of our first mm-VLBI observations $\mathrm{SiO}$ masers were much brighter, with a peak flux of $20 \mathrm{Jy}$, but today their intensity has dropped down by a factor of nearly 40 (in fifteen years), and the peak of the emission is only $\sim 0.5 \mathrm{Jy}$. Given its sensitivity and high resolution ALMA is the only instrument that could provide both the sensitivity and spatial resolution needed to disentangle the question of the absolute position of the $\mathrm{SiO}$ masers.

\section{Observations and Results}

We observed OH 231.8 with ALMA in Band $3(86 \mathrm{GHz})$ as part of the Cycle 4 program (ID: 2016.1.00472.S; PI: J.-F. Desmurs). We used the extended configuration of ALMA (C40-9) to reach the highest spatial resolution available $\left(\sim 0 .{ }^{\prime \prime} 1\right)$. The observation was conducted using 41 $12 \mathrm{~m}$ antennas with baselines ranging from 21 to $14851 \mathrm{~m}$ between $23 \& 24$ September, 2017. The first run failed and the second gave a total time on-source of 21.77 minutes. The precipitable water vapor (PWV) was $0.78 \mathrm{~mm}$ during the observations.

The correlator was configured to simultaneously observed $\mathrm{SiO} v=1 \quad J=2-1$ and $v=2 J=2-1$ with narrow filters of $60 \mathrm{MHz}\left(\sim 210 \mathrm{~km} \mathrm{~s}^{-1}\right.$, the total width of the lines emission covers about $\left.15 \mathrm{~km} \mathrm{~s}^{-1}\right)$ split in 960 channels to reach a high spectral resolution $\left(\sim 0.22 \mathrm{~km} \mathrm{~s}^{-1}\right)$ to properly sample the individual maser components (typically 1 or $2 \mathrm{~km} \mathrm{~s}^{-1}$ ). In addition, we also added two basebands with a broad band width (total aggregate bandwidth $\sim 3.6 \mathrm{GHz}$ ) to detect the continuum and other spectral lines at lower velocity resolution.

For pointing, bandpass and flux calibration we regularly observed quasar J0750+1231 and for phase calibration J0746-1555. The data were calibrated using the Common Astronomy Software Applications (CASA, [14]) package, version 4.7.2. Following the data reduction scripts provided by ALMA we applied the standard bandpass, flux, and gain calibrations. Based on the phase and amplitude variations on calibrators we estimated an absolute flux calibration uncertainty of about $10 \%$. Self-calibration was performed to improve the signal-to-noise ratio. The continuum and $\mathrm{SiO}$ 


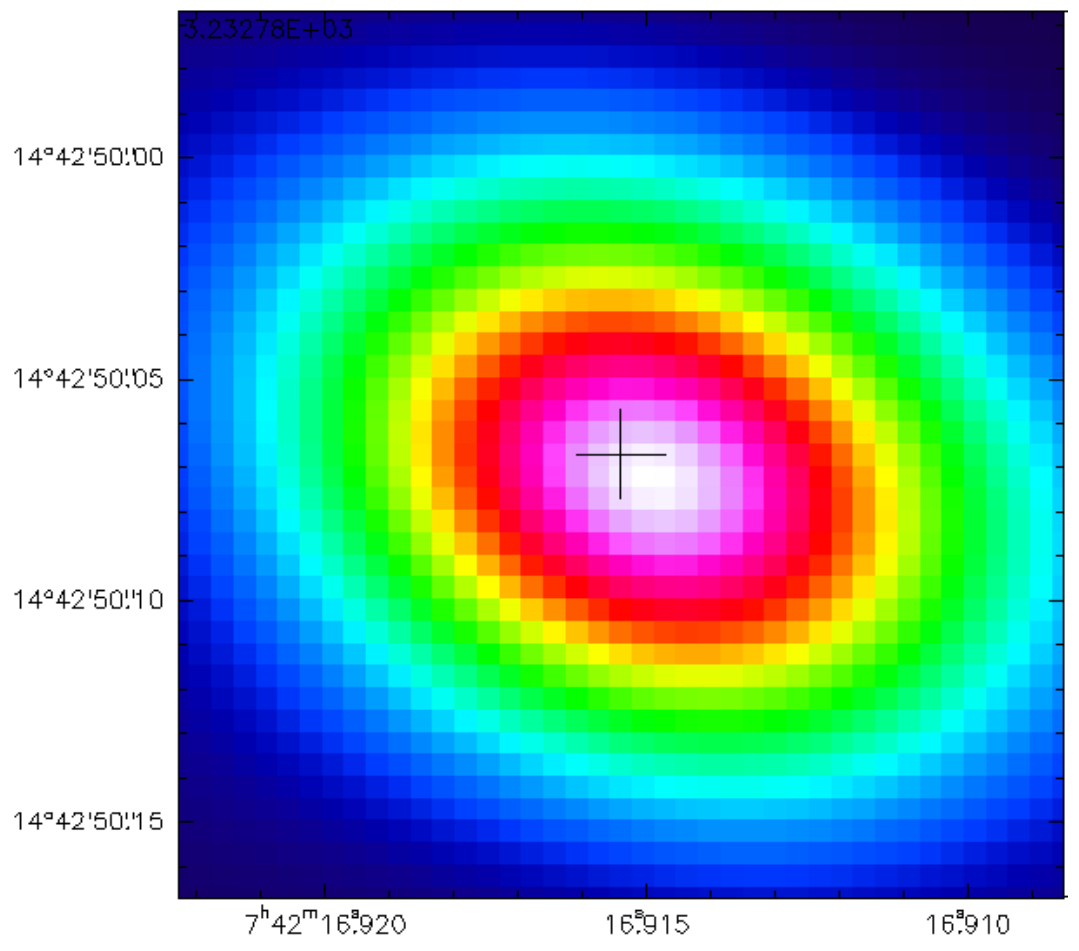

Figure 1: Continuum map at $87 \mathrm{GHz}$ of the $\mathrm{OH} 231.8+4.2$. Beam resolution is $80 x 50 \mathrm{mas}$, $\mathrm{rms}=0.07 \mathrm{mJy}$, the integrated flux density is 3.405 $\pm 0.075 \mathrm{mJy}$. The cross mark the phase center position ICRS 07:42:16.9154 $-14.42 .50 .067$

line images were finally created from the calibrated visibilities using the CASA tclean task with Briggs weighting and the parameter 'robust' set to 0.5 . Maps of $2.5 \times 2.5 \operatorname{arcsec}$ were produced with a restoring beam of $0.1 \times 0.07$ arcsec FWHM (corresponding to a physical scale of 150x105 AU at a distance of $1.54 \mathrm{kpc}$ ). The rms noise level of the image is $\sim 5 \mathrm{mJy} /$ beam per channel. We also produced maps with uniform weighting improving our restoring beam $0.06 \times 0.04 \operatorname{arcsec}$ (setting the parameter "robust=-2" in tclean). Absolute positions can be determined up to an accuracy of about the synthesized HPWB divided by twice the signal-to-noise ratio (SNR); The map of the continuum emission (with a RMS=7.17e-02 mJy) shows a single components slightly elongated in a direction consistent with the axis of the nebulae (see Fig. 1). Modeling the visibility data with the uvmultifit (see [13]) yields a total flux density of $3.5 \pm 0.06 \mathrm{mJy}$ at position $\mathrm{RA}=07: 42: 16.914980 \pm 0.000033 \mathrm{~s}$ $\mathrm{DEC}=-014: 42: 50.072631 \pm 0.000369$ arcsec .

For $\mathrm{SiO}$, apart the thermal line ( $v=0, J=2-1)$, only the $v=1 J=2-1$ maser was detected. Figure 2 shows the two components detected with respectively a blue-shifted peak of about $566.4 \pm 3.8 \mathrm{mJy} / \mathrm{beam}$ and a red-shifted peak of about $1454.9 \pm 6.6 \mathrm{mJy} / \mathrm{beam}$ (value extracted by fitting a Gaussian component with the task uvmultifit) separated by a distance of about $\sim 11$ mas. The absolute position measured for the center of emission of the components is $\mathrm{RA}=07: 42: 16.91522847 \pm 7.7 \times 10^{-7} \mathrm{~s}$ $\mathrm{DEC}=-014: 42: 50.06400164 \pm 1.044 \times 10^{-5}$ arcsec in agreement with the position recently published by [10] (using the KVN). This result places without any ambiguity the $\mathrm{SiO}$ emission in between 


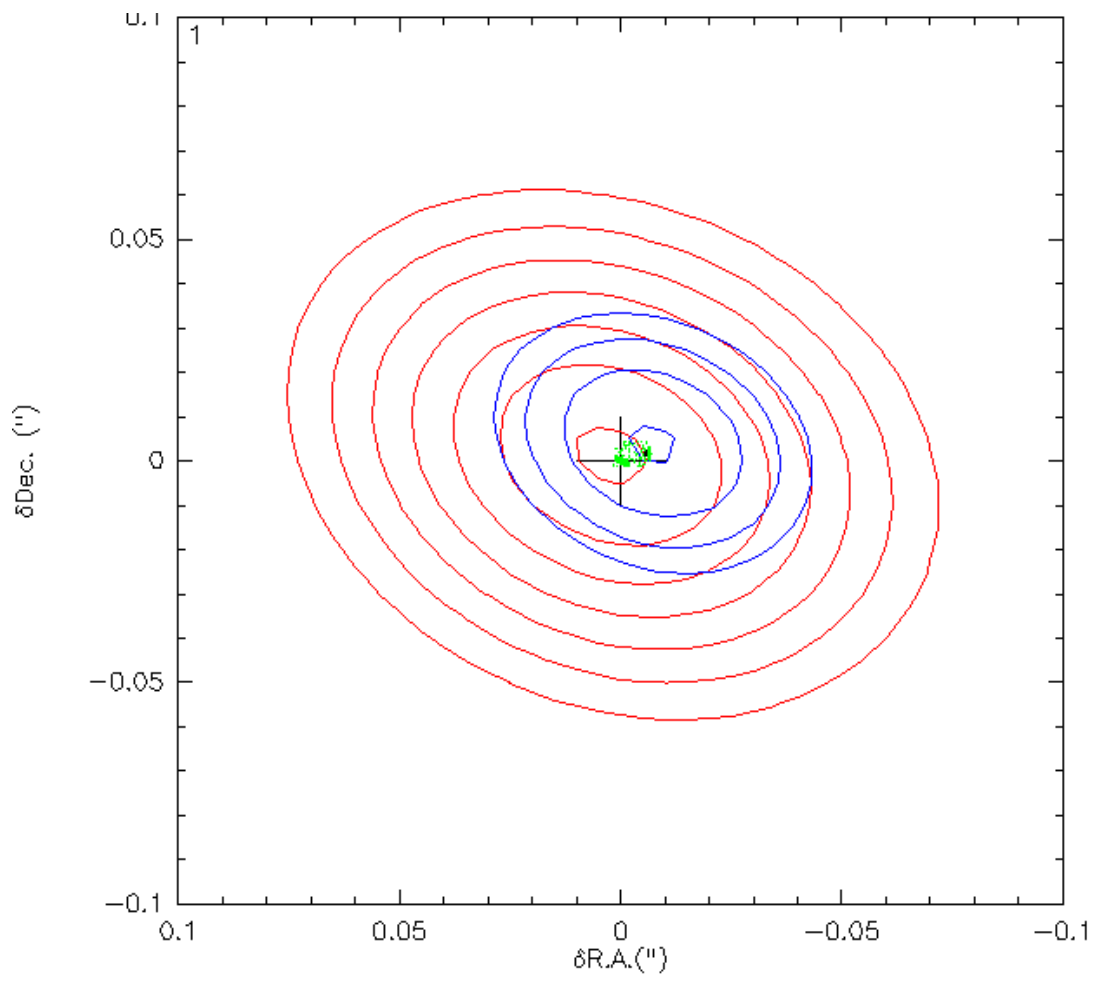

Figure 2: Map of the blue-shifted (in blue) with an integrated flux density of $563.87 \pm 0.44$ mJy and redshifted with an integrated flux density of $1.46081 \pm 0.00086 \mathrm{Jy}$ (red) v=1, J=2-1 SiO emission at $3 \mathrm{~mm}$ detected toward OH 231.8+4.2. The beam resolution is 60x40mas. In green, the VLBA map of SiO v=2, $J=1-0$ observed in 2002 ([17]). The relative position of ALMA and VLBA maps is just indicative. The cross mark the phase center position ICRS 07:42:16.9154 -14:42:50.067

the two areas of water maser emission, suggesting that the binary system is indeed a close one, and that the launching of the jets from the interaction between the two components is indeed possible.

\section{Conclusion}

Using ALMA, we detected and measured the $\mathrm{SiO}$ maser $v=1, J=2-1$ and the thermal $\mathrm{SiO}$ line $(v=0, J=2-1)$. We also detected the continuum emission which traces the binary system, and that is spatially coincident with the $\mathrm{SiO}$ emission. We confirmed the $\mathrm{SiO}$ position tracing the Mira component of the binary system as measured by [8] (and with KVN by [10]). This result solves the discrepancy of the relative position between the $\mathrm{H}_{2} \mathrm{O}$ and the $\mathrm{SiO}$ maser emission and offers a consistent picture of the source: The Mira in the center closely surrounded by $\mathrm{SiO}$ maser emission; farther out but related with the Mira, the $\mathrm{H}_{2} \mathrm{O}$ masers tracing the base of the outflows also detected with the thermal $\mathrm{SiO}$ emission (this observations) and, far away, at larger scale, the $\mathrm{CO}$ outflows.

\section{References}

[1] Alcolea J., Bujarrabal, V., Sánchez Contreras, C., Neri, R. \& Zweigle, J., 2001, A\&A 373, 932. 
[2] Balick \& Frank 2002, ARA \&A 40, 439.

[3] Bujarrabal, V., Alcolea, J., Sánchez Contreras, C., Sahai, R., 2002, A\&A 389, 271.

[4] Bujarrabal, V., Alcolea, J., Soria-Ruiz, R., Planesas, P., Teyssier, D et al., 2012, A\&A 537, 8.

[5] Choi et al. 2012, IAU 287, 407.

[6] Desmurs J.-F., Bujarrabal V., Colomer F. \& Alcolea J., 1999, A\&A 360, 189.

[7] Desmurs J.-F., Sánchez Contreras, C., Bujarrabal, V., Alcolea \& Colomer, F, 2004, Proceedings of the 7th Symposium of the European VLBI Network, p.213-214.

[8] Desmurs J.-F., Alcolea J, Bujarrabal V., Sánchez Contreras, C. \& Colomer F. , 2007, A\&A 468, 189-192.

[9] Diamond P.J., Kemball A.J., Junor W., Zenzus A., Benson J., Dhawan V. et al., 1994, ApJ 430, L61.

[10] Dodson, R., Rioja, M., Bujarrabal, V., et al., 2018, MNRAS 476, 520-527.

[11] Frank, A., Blackman, E.G., 2004, ApJ 614, 737.

[12] Gómez, Y. \& Rodríguez, L.F., 2001, ApJ 557, L109.

[13] Martí-Vidal, I., Vlemmings, W. H. T., Muller, S. \& Casey, S., 2014, A\&A, 563, 136.

[14] McMullin et al. 2007

[15] Riera, A., Raga, A. C. \& Alcolea, J., 2005, RMxAA 41, 147.

[16] Sánchez Contreras C., Bujarrabal V., Neri R., \& Alcolea J., 2000, A\&A 357, 651.

[17] Sánchez Contreras C., Desmurs, J. F., Bujarrabal, V., Alcolea, J., Colomer, F. 2002, A\&A 385, L1.

[18] Sánchez Contreras C., Gil de Paz, A. \& Sahai, R., 2004, ApJ 109, 721.

[19] Sánchez Contreras, C., Velilla Prieto, L., Agúndez, M., Cernicharo, J., Quintana-Lacaci, G, et al., 2015, A\&A 577, 52.

[20] Soker N., 2002, ApJ 568, 726. 